Check for updates

Cite this: RSC Adv., 2018, 8, 32395

\title{
Integrating plasmonic nanostructures with natural photonic architectures in Pd-modified Morpho butterfly wings for sensitive hydrogen gas sensing $†$
}

\author{
Jiaqing He, (D) a Nicolò Simone Villa, ${ }^{\mathrm{b}}$ Zhen Luo, ${ }^{\mathrm{a}}$ Shun An, ${ }^{\mathrm{a}}$ Qingchen Shen, ${ }^{\mathrm{a}}$ \\ Peng Tao, (iD a Chengyi Song, ${ }^{a}$ Jianbo Wu, (D) a Tao Deng (iD *a and Wen Shang*a
}

\begin{abstract}
This work reports a bioinspired three-dimensional (3D) heterogeneous structure for optical hydrogen gas $\left(\mathrm{H}_{2}\right)$ sensing. The structure was fabricated by selective modification of the photonic architectures of Morpho butterfly wing scales with Pd nanostrips. The coupling of the plasmonic mode of the Pd nanostrips with the optical resonant mode of the Morpho biophotonic architectures generated a sharp reflectance peak in the spectra of the Pd-modified butterfly wing, as well as enhancement of light-matter interaction in $\mathrm{Pd}$ nanostrips. Exposure to $\mathrm{H}_{2}$ resulted in a rapid reversible increase in the reflectance of the Pd-modified butterfly wing, and the pronounced response of the reflectance was at the wavelength where the plasmonic mode strongly interplayed with the optical resonant mode. Owing to the synergetic effect of $\mathrm{Pd}$ nanostrips and biophotonic structures, the bioinspired sensor achieved an $\mathrm{H}_{2}$ detection limit of less than $10 \mathrm{ppm}$. Besides, the Pd-modified butterfly wing also exhibited good sensing repeatability. The results suggest that this approach provides a promising optical $\mathrm{H}_{2}$ sensing scheme, which may also offer the potential design of new nanoengineered structures for diverse sensing applications.
\end{abstract}

Received 12th June 2018

Accepted 12th September 2018

DOI: $10.1039 /$ c8ra05046e

rsc.li/rsc-advances

In recent years, intensive researches have been conducted to develop optical $\mathrm{H}_{2}$ sensors with Pd nanostructures, which offer the potential of miniaturization and the fast reaction kinetics originated from the short diffusion lengths for $\mathrm{H}$ atoms. ${ }^{13-20} \mathrm{Pd}$ nanostructure strongly interacts with light and shows intriguing optical phenomenon due to the localized surface plasmon resonance (LSPR). Such LSPR is associated with the resonant excitation of collective oscillations of the free electrons by incident light and can generate large electromagnetic field confinement at nanometer scale. ${ }^{21}$ The position and intensity of the plasmon resonance peak of Pd nanostructure change with the hydrogen-induced changes of volume and dielectric property, which can be used for the readout of the direct nanoplasmonic sensing scheme. ${ }^{22-24}$ Nevertheless, the sensing performance of those plasmonic $\mathrm{H}_{2}$ sensors is restrained from a fundamental limitation, in which the LSPR of Pd nanostructure generally exhibits broad resonance peak owing to the interband electronic transitions..$^{25,26}$ To overcome this problem, several efforts have been devoted to achieve other possible sensing schemes containing Pd nanostructures. For instance, $\mathrm{H}_{2}$ sensor based on perfect absorption in the visible wavelength range was designed, utilizing a coupled plasmonic system that consisted of Pd nanowires arranged on the top of a thick gold (Au) film separated with a spacer layer of $\mathrm{MgF}_{2} \cdot{ }^{27}$ Indirect LSPR sensors were proposed by precisely placing Pd nanoparticles (NPs) in the vicinity of other metallic NPs that possessed superior LSPR properties and acted as optical antennas to
${ }^{a}$ State Key Laboratory of Metal Matrix Composites, School of Materials Science and Engineering, Shanghai Jiao Tong University, 800 Dong Chuan Road, Shanghai 200240, P. R. China.E-mail:dengtao@sjtu.edu.cn; shangwen@sjtu.edu.cn ${ }^{b}$ Department of Chemistry, Materials and Chemical Engineering "Giulio Natta", Politecnico di Milano, Piazza Leonardo Da Vinci 32, 20133, Milano, Italy

$\dagger$ Electronic supplementary information (ESI) available. See DOI: 10.1039/c8ra05046e 
enhance the response of Pd NPs in the stimulation with $\mathrm{H}_{2} \cdot{ }^{28-32}$ Besides, some Au-Pd core-shell nanostructures with various morphologies have also been synthesized through wet chemistry methods in order to take advantage of the local field enhancement of $\mathrm{Au}$ core to improve the optical response to $\mathrm{H}_{2}{ }^{33-37}$

Here we explored a different $\mathrm{H}_{2}$ sensing approach that is based on three-dimensional (3D) photonic architectures of the Morpho butterfly wing scales modified by Pd nanostructures, as shown in Fig. 1. The iridescent scales of Morpho butterfly wing have unique multilayered air-chitin structures and produce a sharp reflectance peak in the blue region of the spectrum. Such structures have been demonstrated in many high-performance optical sensors. ${ }^{38-42}$ We take advantage of the narrow-band resonance and sensitive feature of the photonic crystal to facilitate $\mathrm{H}_{2}$ sensing through incorporating $\mathrm{Pd}$ nanostructures into such biological photonic crystal structures. Specifically, the 3D heterogeneous structures of Morpho butterfly wing containing Pd nanostrips distributed on the edge portion of the lamella layers of the wing scale were generated through physical vapor deposition (PVD) of Pd (Fig. 1). Owing to coupling between the plasmonic mode of Pd nanostrips and the optical resonant mode of the biophotonic nanostructures, the Pdmodified butterfly scales showed a sharp reflection peak, and light-matter interaction in Pd nanostructure was enhanced. We demonstrated that the synergetic effect of Pd nanostrips and biophotonic structures played a role in $\mathrm{H}_{2}$ sensing, which resulted in the sensitive response of the Pd-modified butterfly scales upon exposure to $\mathrm{H}_{2}$. This work should provide some stimulation for the design of sensing platforms that combine plasmonic nanostructures with photonic crystals.

The Morpho sulkowskyi butterfly was chosen as model for the fabrication of $\mathrm{H}_{2}$ sensing platform. As shown in Fig. 2a, the Morpho butterfly wing displays brilliant blue iridescence originated from the elaborate hierarchical photonic structures of the scales. The scales are regularly arranged on the wing surface, as

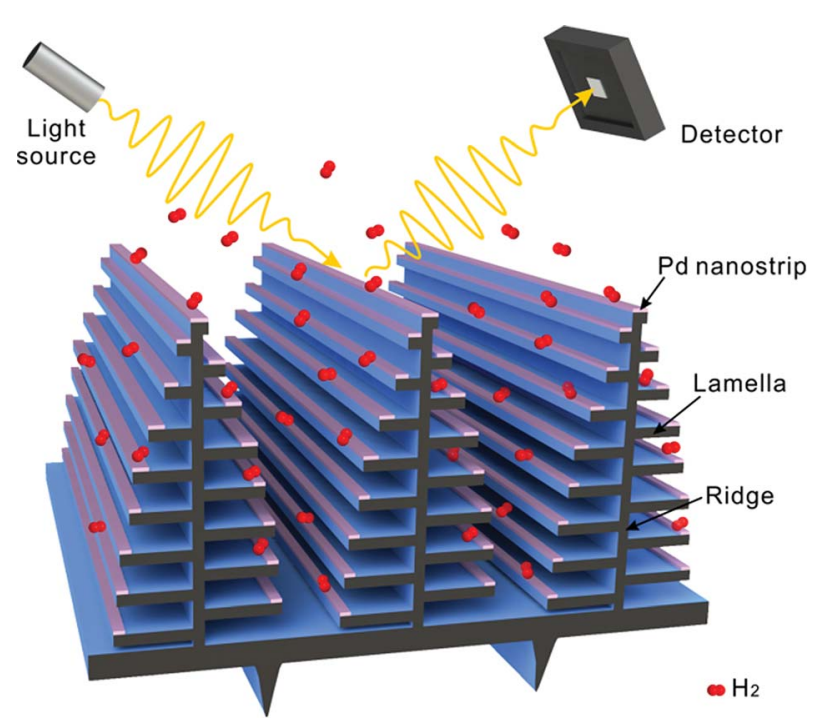

Fig. 1 Schematic illustration showing optical $\mathrm{H}_{2}$ sensing based on the 3D heterogeneous structures that are consisted of Pd nanostrips and the photonic architectures of the Morpho butterfly wing scales.

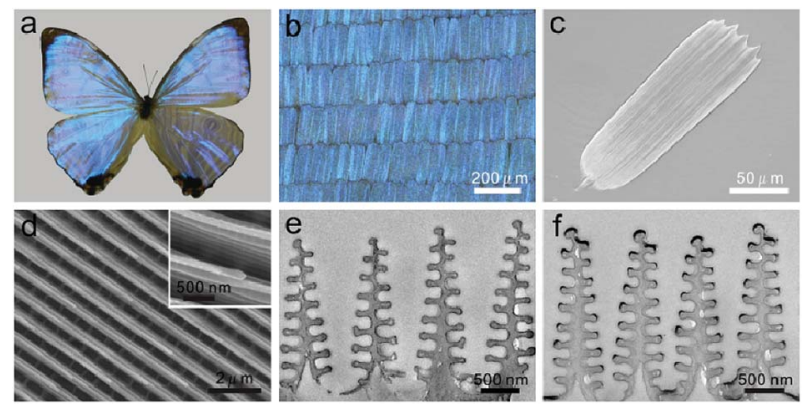

Fig. 2 Morphologies and structures of the Morpho butterfly wing before and after selective modification with $\mathrm{Pd}$. (a) The photo of the Morpho sulkowskyi butterfly. (b) Optical microscopy image of the stacked scales on the wing surface. (c) SEM image of a single butterfly wing scale supported on a silicon substrate. (d) Top SEM view of the photonic architecture of the scale. The inset figure is a high magnification SEM showing the multilayered lamella structures. (e) TEM image of a transverse section of the scale showing ridges with lamella structures. (f) TEM image showing the selectively modified ridges, where the edge of each lamella was coated with Pd nanostrip.

presented in Fig. $2 \mathrm{~b}$. They are $\sim 200 \mu \mathrm{m}$ in length and $\sim 50 \mu \mathrm{m}$ in width (Fig. 2c). On each scale, there are ordered arrays of ridges running along the longitudinal direction and adjacent ridges are connected each other by cross-ribs in the transversal direction, as presented in Fig. 2d. The high magnification scanning electron microscopy (SEM) image in the inset of Fig. 2d shows that the ridges contain multilayered lamellae folds. The details of the multilayered structures are shown by a cross-section transmission electron microscopy (TEM) image of ridges in Fig. 2e, which reveals the Christmas tree-like structures. A stem with width of 50-120 nm stands at the middle of each ridge. Approximately eight lamellae that are separated by air decorate at both sides of the stem, with the width of lamella gradually decreasing from the bottom to the top of the stem. The average thicknesses of lamella and the spacing between the lamella are $\sim 65 \mathrm{~nm}$ and $\sim 150 \mathrm{~nm}$, respectively. Multilayer interference of light from the lamella layers, in combination with the light diffraction from the arrays of the ridges, contributes to the iridescence color appearance of the butterfly wing. ${ }^{42,43}$ We used the PVD method to deposit Pd coating on the butterfly wing structures. Considering the formation of the continuous coating and the response time of the 3D structure, we set the thickness of the Pd coating at $15 \mathrm{~nm}$. During the deposition of Pd, the butterfly wing was placed directly under a Pd source so that the Pd coating was deposited vertically onto the butterfly wing structures. After coating the Pd layer, the edge portions of the lamellae were covered with Pd, resulted in selective modification of photonic structures of butterfly wing scales with Pd nanostrips, as shown in Fig. $2 \mathrm{f}$ and $\mathrm{S} 1 . \dagger$

We investigated the optical reflectance of the Pd-modified butterfly wing at normal incidence. Compared with the reflectance spectra of original scales, the reflectance of Pd-modified butterfly wing scales with a Pd layer of about $15 \mathrm{~nm}$ in thickness exhibited a main peak, which underwent blue shift from $\sim 485 \mathrm{~nm}$ to $\sim 460 \mathrm{~nm}$ and also showed smaller full width at half maximum than the original scales, as shown in Fig. 3a and b. 

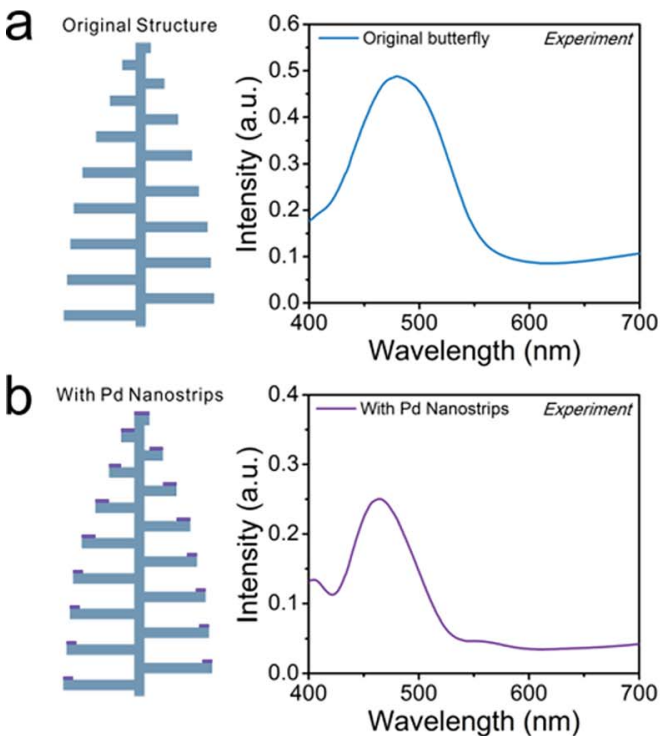
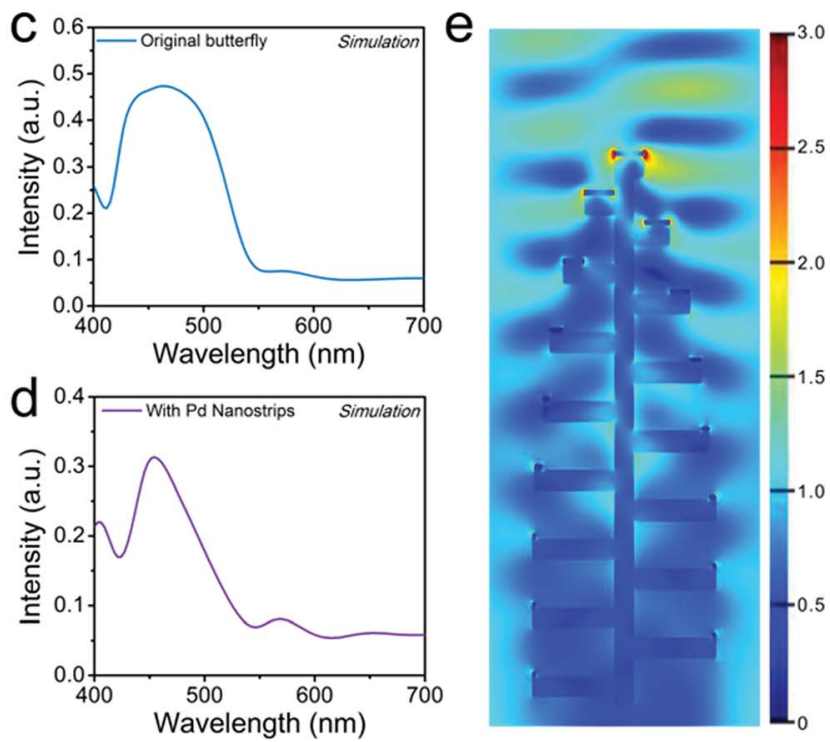

Fig. 3 Optical properties of the original butterfly wing scales and the modified scales with Pd nanostrips. (a) Measured reflectance spectra of the original Morpho butterfly wing. (b) Measured reflectance spectra of the modified butterfly wing scales with Pd nanostrips. (c) Calculated reflectance spectra of the original Morpho butterfly scales. (d) Calculated reflectance spectra of Pd-modified butterfly scales. (e) The simulated electric field distribution at the wavelength of $500 \mathrm{~nm}$ showing the enhanced light-matter interaction in the Pd nanostrips distributed in the butterfly wing structures.

Moreover, decorating of the Morpho scales with Pd nanostrips produced a decrease in the reflectance along with a minor peak at the wavelength near the violet end of the reflection spectra as well as a weak peak at $\sim 560 \mathrm{~nm}$. These spectral changes were most likely due to the interaction of the reflection band originated from butterfly wing structures with the plasmonic absorption from the Pd nanostrips on the wing structures. In order to study this optical interaction, the reflectance spectra for the original butterfly structures and the butterfly structures decorated with Pd nanostrips were calculated through finitedifference time-domain (FDTD) methods, as presented in Fig. $3 \mathrm{c}$ and $\mathrm{d}$, respectively. The simulated reflectance spectra exhibited good matching with the corresponding experimental results. We also performed simulations to separately analyze the plasmonic absorption of Pd nanostrips, as shown in Fig. S2. $\dagger$ The calculated absorption cross-section revealed the broad plasmonic absorption band of Pd nanostrip, which overlaps with the reflection band of butterfly wing structures. Besides, we computed the electric-field distributions of the Pd nanostrips on a planar dielectric substrate (Fig. S3†) and the butterfly wing structures with Pd nanostrips (Fig. 3e) at resonance, respectively. The light was concentrated at the tips of the Pd nanostrips and larger enhancement was observed in the case of the Pd nanostrips distributed in the butterfly wing structures, which indicated that the interaction of light with Pd nanostrips were enhanced by coupling to the optical cavity of Morpho butterfly scales. As such, the plasmonic mode of Pd nanostructures could effectively interplay with resonant mode of the biophotonic nanostructures, which thus was expected to enhance the sensitivity in $\mathrm{H}_{2}$ sensing.

To examine the response of Pd-modified butterfly wing scales to the exposure of $\mathrm{H}_{2}$, the butterfly wing sample was placed in a glass chamber with an optical fiber mounted through the top hatch at room temperature, as shown in Fig. S4. $\dagger$ The chamber was connected to a gas inlet channel for $\mathrm{H}_{2}$ and nitrogen $\left(\mathrm{N}_{2}\right)$ carrier gas that were premixed before flowing into the chamber. The concentration of $\mathrm{H}_{2}$ was regulated through changing the gas flow of $\mathrm{N}_{2}$ and $\mathrm{H}_{2}$ with two mass flow controllers. We recorded the reflectance spectra of the sample at different $\mathrm{H}_{2}$ concentrations. To evaluate the changes in reflectance spectra following $\mathrm{H}_{2}$ exposures, we calculated the relative reflectance $\Delta R(\lambda)$ according to ${ }^{44}$

$$
\Delta R(\lambda)=100 \% \times\left[R(\lambda) / R_{0}(\lambda)\right]
$$

where $R_{0}(\lambda)$ is the spectrum collected from the Pd-modified butterfly wing scales in pure $\mathrm{N}_{2}$ and $R(\lambda)$ is the spectrum collected upon exposure to $\mathrm{H}_{2}$ in $\mathrm{N}_{2}$ carrier gas.

Fig. 4a showed relative reflectance of the Pd-modified butterfly wing scales with respect to different $\mathrm{H}_{2}$ concentrations over a range from $0.001 \%$ to $4 \%$. Exposure to $\mathrm{H}_{2}$ caused the reflectance increase, and the pronounced $\Delta R$ response was over the wavelength region of $400-550 \mathrm{~nm}$, which took place in the same wavelength range of the strong interaction between the plasmonic mode of Pd nanostructures and resonant mode of the biophotonic nanostructures. The reflectance of the original Morpho butterfly wing scales with respect to different $\mathrm{H}_{2}$ concentrations over a range from $0.1 \%$ to $4 \%$ also was measured, which suggested that there was no obvious response (Fig. S5 $\dagger$ ). In the presence of $\mathrm{H}_{2}$, the Pd nanostrips dissociated $\mathrm{H}_{2}$ molecules and absorbed the $\mathrm{H}$ atoms to form Pd hydride, typically accompanied by lattice expansion and the change of dielectric properties to less metallic. As a result, the plasmonic absorption of the Pd nanostrip was altered and the spectral variations of the heterogeneous structures were induced. The 

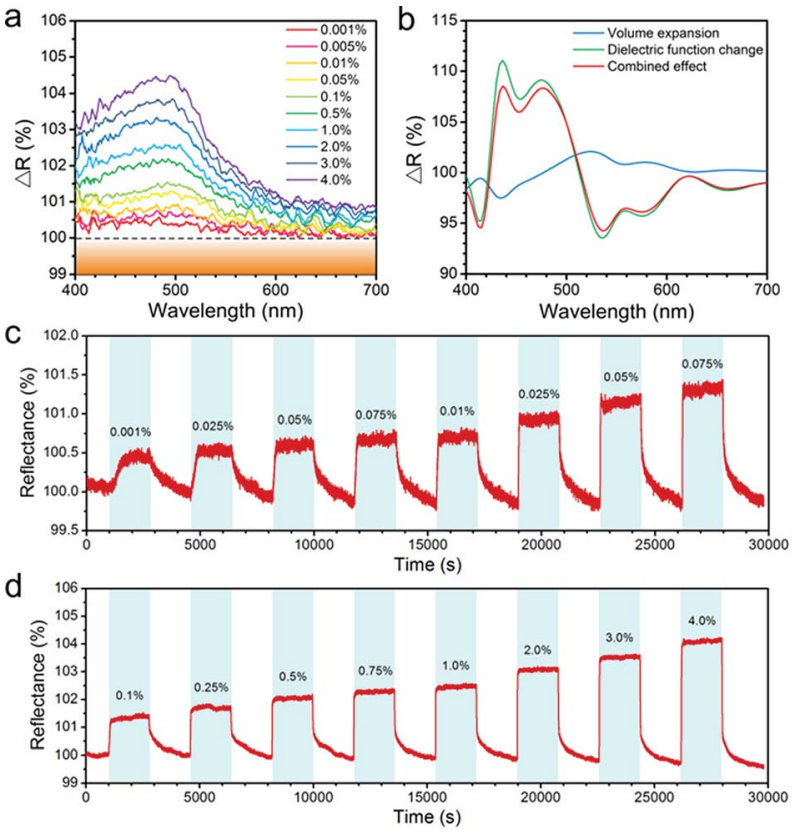

Fig. 4 Response of the Pd-modified photonic architectures to $\mathrm{H}_{2}$ gas. (a) Relative reflectance measured in different $\mathrm{H}_{2}$ concentrations. (b) The simulated relative reflectance of the $\mathrm{Pd}$-modified butterfly scales due to expansion and change in dielectric function when $\mathrm{Pd}$ is converted into $\beta-\mathrm{PdH}_{x}$. (c) Normalized reflectance change at wavelength of $500 \mathrm{~nm}$ in different $\mathrm{H}_{2}$ concentrations showing a detection limit below $10 \mathrm{ppm}$. (d) Normalized reflectance change at wavelength of $500 \mathrm{~nm}$ in different $\mathrm{H}_{2}$ concentrations from $0.1 \%$ to $4 \%$.

increase in the reflectance response of the Pd-modified butterfly wing was observed with the increase in $\mathrm{H}_{2}$ concentration, which was attributed to the larger change of dielectric function and volume expansion.

To further understand the sensing mechanism, we carried out simulations to compare the reflectance of Pd-modified butterfly scales as well as the Pd nanostrips on a planar dielectric substrate before and after $\mathrm{H}_{2}$ uptake, as shown in Fig. $4 \mathrm{~b}$ and S6. $\dagger$ For simplicity, we assumed that the Pd was completely converted into $\beta$-phase $\left(\beta-\mathrm{PdH}_{x}\right)$ after the exposure of $\mathrm{H}_{2}$ gas. In the simulations, we examined the contributions of two factors: volume expansion and the change in dielectric function. The volume expansion of Pd nanostrips alone led to a little decrease in the $\Delta R(\lambda)$ spectra of the heterogeneous structures over the short-wavelength range and slight increase over the long-wavelength range. The change in dielectric function led to a clear increase in the $\Delta R(\lambda)$ spectra over the $430-510 \mathrm{~nm}$. The calculated results of the combined effects of volume expansion and the change in dielectric function suggested that the change of dielectric function has more influence on the optical response of the Pd-modified biophotonic structures. The calculated $\Delta R(\lambda)$ spectra in Fig. $4 \mathrm{~b}$ are in general agreement with the experimental spectra (Fig. 4a). The pronounced feature in calculated and experimental spectra was the increase in the $\Delta R(\lambda)$ spectra over the wavelength range of the strong interaction between the plasmonic mode and the resonant mode of the biophotonic nanostructures.

The dynamic change of reflectance for the Pd-modified butterfly wing sample at the wavelength of $500 \mathrm{~nm}$ were shown in Fig. $4 \mathrm{c}$ and d. The optical response clearly showed the dependence on the $\mathrm{H}_{2}$ concentration. For comparison, the temporal reflectance was normalized to the reflectance of the sample in pure $\mathrm{N}_{2}$. As the concentration of $\mathrm{H}_{2}$ increased, the normalized reflectance at wavelength of $500 \mathrm{~nm}$ increased, and the sensor exhibited a lowest detectable response at concentrations of $10 \mathrm{ppm}$. The low noise level relative to the response indicated a detection limit less than $10 \mathrm{ppm}$, which is among the lowest detection limit for optical $\mathrm{H}_{2}$ sensing based on plasmonic Pd nanostructures..$^{13,19,30}$ The high sensitivity of the Pd-modified butterfly wing scales mainly arose from the synergetic effect between Pd nanostrips and biophotonic structures.

Furthermore, the reflectance change at the wavelength of $500 \mathrm{~nm}$ as a function of $\mathrm{H}_{2}$ concentration in the range from $0.001 \%$ to $4 \%$ suggested a positive relationship (Fig. $5 \mathrm{a}$ ). It is worth to note that a linear relationship between the reflectance change and the $\mathrm{H}_{2}$ concentrations was observed within the high concentration range from $0.75 \%$ to $4 \%$ (inset of Fig. $5 a$ ), which is approximately corresponding to the regimes of mixed $\alpha+\beta$-phase and the $\beta$-phase Pd hydride., ${ }^{4,30}$ We also measured the response time of the Pd-modified butterfly wing scales at different $\mathrm{H}_{2}$ concentrations (Fig. 5b). The response time was defined as the time needed to reach $90 \%$ of the equilibrium $\Delta R$. As depicted in Fig. 5b, when $\mathrm{H}_{2}$ concentration increased, the response time decreased first, and then gradually became relatively stable. The observed response time is within the range of response time reported so far. ${ }^{27,33}$ The sensing speed could be further improved by using different materials such as the Pd alloys. ${ }^{45}$

In addition, we monitored the optical response of our sensor during five on/off $\mathrm{H}_{2}$ cycles at the concentration of $0.5 \%$ and $1 \%$, respectively. The temporal response of normalized reflectance at the wavelength of $500 \mathrm{~nm}$ was shown in Fig. 5c. The Pdmodified butterfly wing scales exhibited a rather consistent
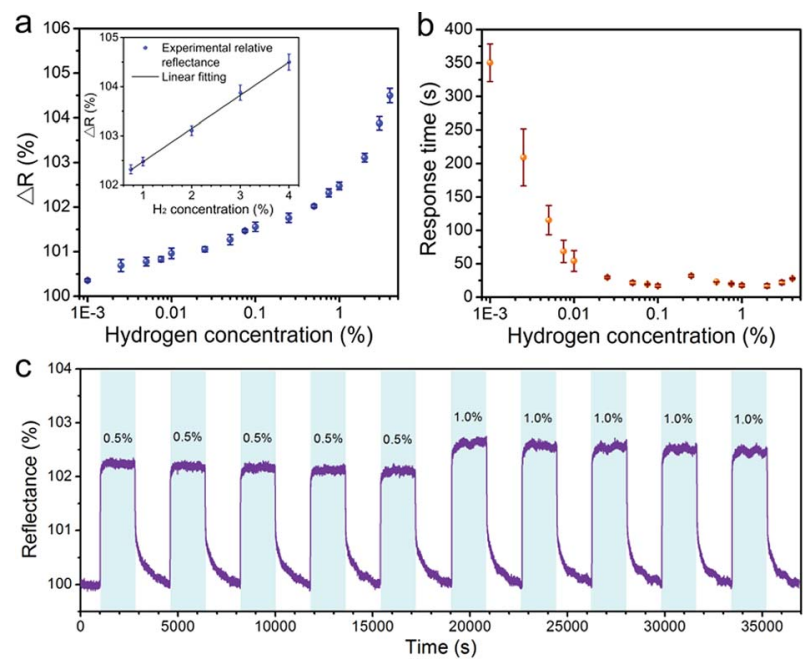

Fig. 5 Sensing performance of the Pd-modified Morpho butterfly scales to $\mathrm{H}_{2}$. (a) The plot showing relative reflectance at wavelength of $500 \mathrm{~nm}$ for different $\mathrm{H}_{2}$ concentrations. The inset figure shows a good linear relation between the relative reflectance and the $\mathrm{H}_{2}$ concentration at the range of $0.75-4 \%$. (b) The response time for different $\mathrm{H}_{2}$ concentrations. (c) Five cycles of $\mathrm{H}_{2}$ exposure for concentrations of $0.5 \%$ and $1 \%$. 
response for each repeated cycle, which suggested a good repeatability and stability of $\mathrm{H}_{2}$ sensing.

For investigation of the selective response of the Pd-modified Morpho butterfly wing scales, the relative reflectances upon exposure to $\mathrm{H}_{2}$ and several potential interfering gases, including $\mathrm{O}_{2}, \mathrm{CO}_{2}$, and $\mathrm{CH}_{4}$ were compared, as shown in Fig. S7. $\uparrow$ The responses from the interfering gases were much less than that observed for $\mathrm{H}_{2}$ at similar concentration, indicating that the $3 \mathrm{D}$ heterogeneous structure has a little crosssensitivity to these interfering gases.

\section{Conclusions}

In summary, we presented a bioinspired 3D heterogeneous structure that was achieved by selectively modification of Morpho butterfly wing scales with $\mathrm{Pd}$ for sensitive $\mathrm{H}_{2}$ sensing at room temperature. The plasmonic mode in Pd nanostrips was effectively coupled with the optical resonant mode of the biophotonic nanostructures in the as-fabricated Pd-modified butterfly wing scales, which produced a sharp major peak in reflectance spectra and yielded an enhancement of light interaction with Pd nanostrips. Both the experiments and simulations demonstrated that the pronounced response of the reflectance spectra to the exposure of $\mathrm{H}_{2}$ was occurred over the wavelength range of the strong interplay between the plasmonic mode and the optical resonant mode. Due to the synergetic effect of Pd nanostrips and biophotonic structures, the Pd-modified butterfly scales exhibited high sensitivity to $\mathrm{H}_{2}$ with a detection limit below $10 \mathrm{ppm}$. Besides, the fabricated optical $\mathrm{H}_{2}$ sensor also showed fast response and good repeatability. The bioinspired $\mathrm{H}_{2}$ sensing system, which is based on the combination of plasmonic nanostructures with photonic crystal structures, not only provides a promising approach for high-performance optical $\mathrm{H}_{2}$ sensor, but also offers a strategy of developing future nanoengineered structures for various sensing applications.

\section{Conflicts of interest}

There are no conflicts to declare.

\section{Acknowledgements}

We are grateful to the financial support from National Key R\&D Program of China (2016YFB0402100), National Natural Science Foundation of China (Grant No: 51521004, 51420105009, 51403127, 21401129), the 111 Project (Grant No: B16032) and "Chen Guang" project from Shanghai Municipal Education Commission. Thanks Ge Wang and Yanhua Zhu from the Instrumental Analysis Center of Shanghai Jiao Tong University for help with the TEM samples.

\section{References}

1 G. Cipriani, V. Di Dio, F. Genduso, D. La Cascia, R. Liga, R. Miceli and G. Ricco Galluzzo, Int. J. Hydrogen Energy, 2014, 39, 8482-8494.
2 S. Singh, S. Jain, V. Ps, A. K. Tiwari, M. R. Nouni, J. K. Pandey and S. Goel, Renewable Sustainable Energy Rev., 2015, 51, 623633.

3 T. Hübert, L. Boon-Brett, G. Black and U. Banach, Sens. Actuators, B, 2011, 157, 329-352.

4 F. D. Manchester, A. San-Martin and J. M. Pitre, J. Phase Equilib., 1994, 15, 62-83.

5 M. E. Nasir, W. Dickson, G. A. Wurtz, W. P. Wardley and A. V. Zayats, Adv. Mater., 2014, 26, 3532-3537.

6 F. Favier, E. C. Walter, M. P. Zach, T. Benter and R. M. Penner, Science, 2001, 293, 2227-2231.

7 Y. T. Pan, X. Yin, K. S. Kwok and H. Yang, Nano Lett., 2014, 14, 5953-5959.

8 X. Li, M. Le Thai, R. K. Dutta, S. Qiao, G. T. Chandran and R. M. Penner, ACS Sens., 2017, 2, 282-289.

9 M. Chen, P. Mao, Y. Qin, J. Wang, B. Xie, X. Wang, D. Han, G. H. Wang, F. Song, M. Han, J. M. Liu and G. Wang, ACS Appl. Mater. Interfaces, 2017, 9, 27193-27201.

10 Z. Zhao, Y. Sevryugina, M. A. Carpenter, D. Welch and H. Xia, Anal. Chem., 2004, 76, 6321-6326.

11 Y.-n. Zhang, H. Peng, X. Qian, Y. Zhang, G. An and Y. Zhao, Sens. Actuators, B, 2017, 244, 393-416.

12 Y. Shen, X. She and C. Jin, ACS Photonics, 2018, 5, 1334-1342. 13 I. Zoric, E. M. Larsson, B. Kasemo and C. Langhammer, Adv. Mater., 2010, 22, 4628-4633.

14 T. Shegai, P. Johansson, C. Langhammer and M. Kall, Nano Lett., 2012, 12, 2464-2469.

15 M. A. Poyli, V. M. Silkin, I. P. Chernov, P. M. Echenique, R. D. Muino and J. Aizpurua, J. Phys. Chem. Lett., 2012, 3, 2556-2561.

16 C. Wadell, S. Syrenova and C. Langhammer, ACS Nano, 2014, 8, 11925-11940.

17 K. Ikeda, S. Uchiyama, M. Takase and K. Murakoshi, ACS Photonics, 2015, 2, 66-72.

18 F. A. Nugroho, B. Iandolo, J. B. Wagner and C. Langhammer, ACS Nano, 2016, 10, 2871-2879.

19 K. C. Ng, F.-C. Lin, P.-W. Yang, Y.-C. Chuang, C.-K. Chang, A.-H. Yeh, C.-S. Kuo, C.-R. Kao, C.-C. Liu, U. S. Jeng, J.-S. Huang and C.-H. Kuo, Chem. Mater., 2017, 30, 204-213.

20 M. Matuschek, D. P. Singh, H. H. Jeong, M. Nesterov, T. Weiss, P. Fischer, F. Neubrech and N. Liu, Small, 2018, 14, 1702990.

21 C. Langhammer, Z. Yuan, I. Zoric and B. Kasemo, Nano Lett., 2006, 6, 833-838.

22 C. Langhammer, I. Zoric, B. Kasemo and B. M. Clemens, Nano Lett., 2007, 7, 3122-3127.

23 C. Langhammer, V. P. Zhdanov, I. Zoric and B. Kasemo, Phys. Rev. Lett., 2010, 104, 135502.

24 F. Yang, S. C. Kung, M. Cheng, J. C. Hemminger and R. M. Penner, ACS Nano, 2010, 4, 5233-5244.

25 J. M. Sanz, D. Ortiz, R. Alcaraz de la Osa, J. M. Saiz, F. González, A. S. Brown, M. Losurdo, H. O. Everitt and F. Moreno, J. Phys. Chem. C, 2013, 117, 19606-19615.

26 T. Pakizeh, C. Langhammer, I. Zoric, P. Apell and M. Kall, Nano Lett., 2009, 9, 882-886.

27 A. Tittl, P. Mai, R. Taubert, D. Dregely, N. Liu and H. Giessen, Nano Lett., 2011, 11, 4366-4369. 
28 T. Shegai and C. Langhammer, Adv. Mater., 2011, 23, 44094414.

29 C. Langhammer, E. M. Larsson, B. Kasemo and I. Zoric, Nano Lett., 2010, 10, 3529-3538.

30 A. Yang, M. D. Huntington, M. F. Cardinal, S. S. Masango, R. P. Van Duyne and T. W. Odom, ACS Nano, 2014, 8, 7639-7647.

31 N. Liu, M. L. Tang, M. Hentschel, H. Giessen and A. P. Alivisatos, Nat. Mater., 2011, 10, 631-636.

32 C. Wadell and C. Langhammer, Nanoscale, 2015, 7, 1096310969.

33 C. Y. Chiu and M. H. Huang, Angew. Chem., Int. Ed., 2013, 52, 12709-12713.

34 R. Jiang, F. Qin, Q. Ruan, J. Wang and C. Jin, Adv. Funct. Mater., 2014, 24, 7328-7337.

35 H. K. Yip, X. Zhu, X. Zhuo, R. Jiang, Z. Yang and J. Wang, Adv. Opt. Mater., 2017, 5, 1700740.

36 S. Rodal-Cedeira, V. Montes-García, L. Polavarapu, D. M. Solís, H. Heidari, A. La Porta, M. Angiola, A. Martucci, J. M. Taboada, F. Obelleiro, S. Bals, J. PérezJuste and I. Pastoriza-Santos, Chem. Mater., 2016, 28, 91699180.

37 M. L. Tang, N. Liu, J. A. Dionne and A. P. Alivisatos, J. Am. Chem. Soc., 2011, 133, 13220-13223.
38 X. Yang, Z. Peng, H. Zuo, T. Shi and G. Liao, Sens. Actuators, A, 2011, 167, 367-373.

39 R. A. Potyrailo, T. A. Starkey, P. Vukusic, H. Ghiradella, M. Vasudev, T. Bunning, R. R. Naik, Z. Tang, M. Larsen, T. Deng, S. Zhong, M. Palacios, J. C. Grande, G. Zorn, G. Goddard and S. Zalubovsky, Proc. Natl. Acad. Sci. U. S. A., 2013, 110, 15567-15572.

40 F. Zhang, Q. Shen, X. Shi, S. Li, W. Wang, Z. Luo, G. He, P. Zhang, P. Tao, C. Song, W. Zhang, D. Zhang, T. Deng and W. Shang, Adv. Mater., 2015, 27, 1077-1082.

41 D. Xu, H. Yu, Q. Xu, G. Xu and K. Wang, ACS Appl. Mater. Interfaces, 2015, 7, 8750-8756.

42 A. D. Pris, Y. Utturkar, C. Surman, W. G. Morris, A. Vert, S. Zalyubovskiy, T. Deng, H. T. Ghiradella and R. A. Potyrailo, Nat. Photonics, 2012, 6, 195-200.

43 P. Vukusic, J. R. Sambles, C. R. Lawrence and R. J. Wootton, Proc. R. Soc. London, Ser. B, 1999, 266, 1403-1411.

44 R. A. Potyrailo, H. Ghiradella, A. Vertiatchikh, K. Dovidenko, J. R. Cournoyer and E. Olson, Nat. Photonics, 2007, 1, 123128.

45 C. Wadell, F. A. Nugroho, E. Lidstrom, B. Iandolo, J. B. Wagner and C. Langhammer, Nano Lett., 2015, 15, 3563-3570. 